\title{
Emergence of self-organized amoeboid movement in a multi-agent approximation of Physarum polycephalum
}

\author{
Jeff Jones and Andrew Adamatzky \\ Centre for Unconventional Computing, University of the West of England, Coldharbour Lane, \\ Bristol BS16 1QY, UK \\ E-mail: jeff.jones@uwe.ac.uk and andrew.adamatzky@uwe.ac.uk
}

Received 26 September 2011

Accepted for publication 3 January 2012

Published DD MM 2012

Online at stacks.iop.org/BB/7/000000

\begin{abstract}
The giant single-celled slime mould Physarum polycephalum exhibits complex morphological adaptation and amoeboid movement as it forages for food and may be seen as a minimal example of complex robotic behaviour. Swarm computation has previously been used to explore how spatio-temporal complexity can emerge from, and be distributed within, simple component parts and their interactions. Using a particle-based swarm approach we explore the question of how to generate collective amoeboid movement from simple non-oscillatory component parts in a model of $P$. polycephalum. The model collective behaves as a cohesive and deformable virtual material, approximating the local coupling within the plasmodium matrix. The collective generates de-novo and complex oscillatory patterns from simple local interactions. The origin of this motor behaviour distributed within the collective rendering is morphologically adaptive, amenable to external influence and robust to simulated environmental insult. We show how to gain external influence over the collective movement by simulated chemo-attraction (pulling towards nutrient stimuli) and simulated light irradiation hazards (pushing from stimuli). The amorphous and distributed properties of the collective are demonstrated by cleaving it into two independent entities and fusing two separate entities to form a single device, thus enabling it to traverse narrow, separate or tortuous paths. We conclude by summarizing the contribution of the model to swarm-based robotics and soft-bodied modular robotics and discuss the future potential of such material approaches to the field.
\end{abstract}

Q1 (Some figures may appear in colour only in the online journal)

\section{Introduction}

Classical robotics approaches typically connect separate sensory, control and locomotion systems, using complex parts with little redundancy. Nature inspired robotics takes inspiration from aggregate populations, collective transport, segmentation of component parts and soft-bodied motion in living systems. Swarm approaches to nature inspired computing seek to elucidate the sensory mechanisms and individual interactions which generate the complexity patterning and movement seen at very different scales in natural systems including car traffic dynamics (Helbing
2001), human walking patterns (Helbing et al 1998, 2001), flocking and schooling (Reynolds 1987), collective insect movement (Buhl et al 2006, Kube and Bonabeau 2000) and bacterial patterning (Matsushita et al 1999, Ben-Jacob 2003). In all these examples there is a population of entities in space, coupled by sensory information. The different sensory coupling mechanisms between individuals in these apparently disparate systems has also been abstracted at a minimal level of self-propelled particles to find common mechanisms (Vicsek et al 1995).

Swarm approaches typically approximate collective movement at the population level. Distributed generation 
and control of movement also occurs at the individual level (Kennedy et al 2001). Specific examples include flagellated movement of bacteria (Zhang et al 2009), eukaryotes (Dreyfus et al 2005), ciliated transport (Suh et al 2000), peristaltic propulsion (Trimmer et al 2006, Saga and Nakamura 2004) and amoeboid movement (Yokoi et al 2003, Umedachi et al 2009). Many organisms make use of segmentation, employing identical or similar sub-units to form their body plan and generate structure and movement. Such modularity is attractive to computer science and robotics but presents challenges in terms of how to configure, communicate between and control the devices. Population-based approaches have also proved useful in tackling some of these issues. Internal propagation of simulated hormone signals was used to control gait patterns in simulated caterpillar-like modular robots (Salemi et al 2001). Reconfiguration stimulated by crystalline mechanical transformation within a population of automata was demonstrated in Rus and Vona (2000) and future possibilities (and difficulties) in communicating between entities at larger scales with massive populations were discussed in Goldstein et al (2009). The exploitation of external environmental attractants ('seeds' and 'scents') was used to affect the reconfiguration of a simulated modular robotic system composed of simple finite state machines for the creation of specific manipulation structures (Bojinov et al 2002), and pre-desired shapes (Stoy 2006).

Progress in biologically inspired robotics has also been made by considering the use of even simpler structures which straddle the boundary of non-living physical materials and living organisms including those acting as biological fibres and membranes (Zhang 2003), lipid self assembly in terms of networks (Lobovkina et al 2008), pseudopodium-like membrane extension (Lobovkina et al 2009) and even those exhibiting simple chemotaxis responses (Lagzi et al ????). Some engineering and biological insights have already been gained by studying the structure and function of what might be termed 'semi-biological' materials and the complex behaviour seen in such minimal examples raises questions about the lower bounds necessary for the emergence of apparently intelligent behaviour.

An ideal hypothetical candidate for a biological machine would be an organism which is capable of the complex sensory integration, movement and adaptation of a living organism, yet which is also composed of a relatively simple material that is amenable to simple understanding and control of its properties. We suggest that the myxomycete organism, the true slime mould Physarum polycephalum, is a suitable candidate organism which meets both criteria; i.e. it is a complex organism, but which is composed of relatively simple materials. A giant single-celled organism, P. polycephalum is an attractive biological candidate medium for emergent motive force because the basic physical mechanism during the plasmodium stage of its life cycle is a self-organized system of oscillatory contractile activity which is used in the pumping and distribution of nutrients within its internal transport network. The organism is remarkable in that the control of the oscillatory behaviour is distributed throughout the almost homogeneous medium and is highly redundant, having no critical or unique components.
The plasmodium is amorphous in shape and ranges from the microscopic scale to up to many square metres in size. It is a single cell syncytium formed by repeated nuclear division, comprised of a sponge-like actomyosin complex co-occurring in two physical phases. The gel phase is a dense matrix subject to spontaneous contraction and relaxation, under the influence of changing concentrations of intracellular chemicals. The protoplasmic sol phase is transported through the plasmodium by the force generated by the oscillatory contractions within the gel matrix. Protoplasmic flux, and thus the behaviour of the organism, is affected by changes in pressure, temperature, space availability, chemoattractant stimuli and illumination (Carlile 1970, Durham and Ridgway 1976, Kishimoto 1958, Nakagaki et al 1996, 2000b, Takamatsu et al 2000, Ueda et al 1975). The $P$. polycephalum plasmodium can thus be regarded as a complex functional material capable of both sensory and motor behaviour. Indeed P. polycephalum has been described as a membrane bound reaction-diffusion system in reference to both the complex interactions within the plasmodium and the rich computational potential afforded by its material properties (Adamatzky et al 2008). The study of the computational potential of the $P$. polycephalum plasmodium was initiated by Nakagaki et al (2000a) who found that the plasmodium could solve simple maze puzzles. This research has been extended and the plasmodium has demonstrated its performance in, for example, path planning and plane division problems (Shirakawa and Gunji 2010, Shirakawa et al 2009), spanning trees and proximity graphs (Adamatzky 2007, 2008), simple memory effects (Saigusa et al 2008), the implementation of individual logic gates (Tsuda et al 2004) and $P$. polycephalum inspired models of simple adding circuits (Jones and Adamatzky 2010).

From a robotics perspective it was shown that by its adaptation to changing conditions within its environment, the plasmodium may be considered as a prototype micromechanical manipulation system, capable of simple and programmable robotic actions including the manipulation (pushing and pulling) of small scale objects (Adamatzky and Jones 2008), transport and mixing of substances (Adamatzky 2010c) and as a guidance mechanism in a biologicalmechanical hybrid approach where the response of the plasmodium to light irradiation was used to provide feedback control to a robotic system (Tsuda et al 2007). A $P$. polycephalum inspired approach to amoeboid robotics was demonstrated by Umedachi et al (2009) in which an external ring of coupled oscillators, each connected to passive and tuneable springs, was coupled to a fluid filled inner bladder. The compression of the peripheral springs mimicked the gel contractile phase and the flux of sol within the plasmodium was approximated by the coupled transmission of water pressure to inactive (softer) springs, thus deflecting the peripheral shape of the robot. The resulting movement exhibited flexible behaviour and amoeboid movement.

This paper is also motivated by the complex sensory and foraging behaviour of $P$. polycephalum; however it takes a different approach from that of Umedachi et al. Instead of trying to build an amoeboid robot with pre-existing oscillatory components, we investigate how 


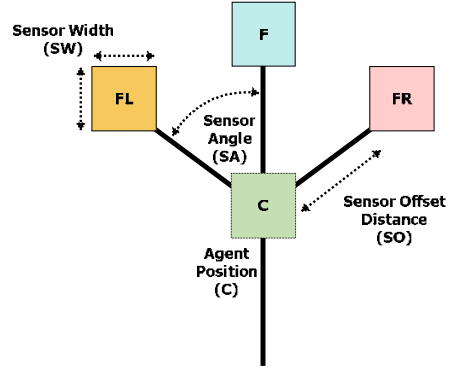

(a)

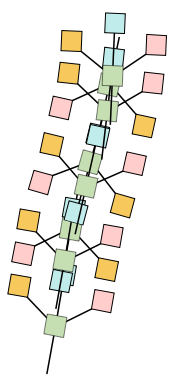

(c)

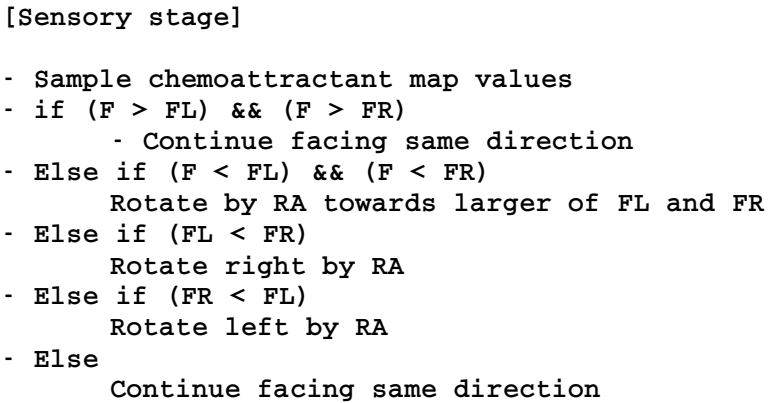

(b)

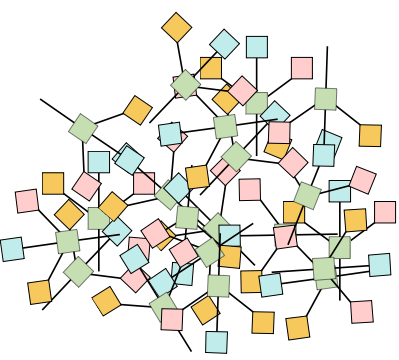

$(d)$

Figure 1. Agent particle morphology, algorithm and aggregation types. (a) Schematic illustration of agent particle showing central position and offset sensors. $(b)$ Pseudocode of agent sensory algorithm to align with strongest source from the forward biased sensors. (c) Schematic illustration of agent particles in transport network configuration. (d) Schematic illustration of agent particles within simulated plasmodial sheet.

oscillatory behaviour may emerge from the local interactions between simple component parts to generate self-organized amoeboid movement. The method uses a swarm-based, or multi-agent, population exploiting self-organization to behave as a collective virtual material. We demonstrate the emergence of the oscillatory phenomena within the material, its utilization for collective, amorphous and controllable amoeboid movement, and mechanisms for external control of the material by simulated chemoattraction and repulsion by light hazards. We conclude by discussing some novel properties of the approach within the context of existing research into swarm robotics and modular robotics.

\section{Methods}

To investigate the use of emergent oscillatory phenomena for collective movement we employ a simple extension to the particle approximation of $P$. polycephalum network adaptation in Jones (2010b) which was shown to generate dynamical emergent transport networks. In this approach a plasmodium is composed of a population of mobile particles with very simple behaviours, residing within a 2D diffusive environment. A discrete 2D lattice (where the features of the environment arena are mapped to greyscale values in a $2 \mathrm{D}$ image) stores particle positions and also the concentration of a local factor which we refer to generically as chemoattractant. The 'chemoattractant' factor actually represents the hypothetical flux of sol within the plasmodium. Free particle movement represents the sol phase of the plasmodium. Particle positions represent the fixed gel structure (i.e. global pattern) of the plasmodium. Particles act independently and iteration of the particle population is performed randomly to avoid introducing any artefacts from sequential ordering. Particle behaviour is divided into two distinct stages, the sensory stage and the motor stage. In the sensory stage, the particles sample their local environment using three forward biased sensors whose angle from the forward position (the sensor angle parameter, SA) and distance (sensor offset, SO) may be parametrically adjusted (figure 1(a)). The offset sensors represent the overlapping and intertwining filaments within the transport networks and plasmodium, generating local coupling of sensory inputs and movement to form networks of particles (figure 1(c)) and sheets of particles (figure $1(d)$ ). The SO distance is measured in pixels and a minimum distance of 3 pixels is required for strong local coupling to occur. The coupling effect increases as SO increases.

During the sensory stage each particle changes its orientation to rotate (via the parameter rotation angle, RA) towards the strongest local source of chemoattractant (figure $1(b)$ ). After the sensory stage, each particle executes the motor stage and attempts to move forwards in its current orientation (an angle from $0^{\circ}-360^{\circ}$ ) by a single pixel. Each lattice site may only store a single particle and-criticallyparticles deposit chemoattractant into the lattice only in the event of a successful forward movement (figure 2(a)). If the next chosen site is already occupied by another particle the default (non-oscillatory) behaviour is to abandon the move, remain in the current position and select a new random direction (figure $2(b)$ ).

Diffusion of the collective chemoattractant signal is achieved via a simple $3 \times 3$ mean filter kernel with a damping parameter (set to 0.07 ) to limit the diffusion distance of 


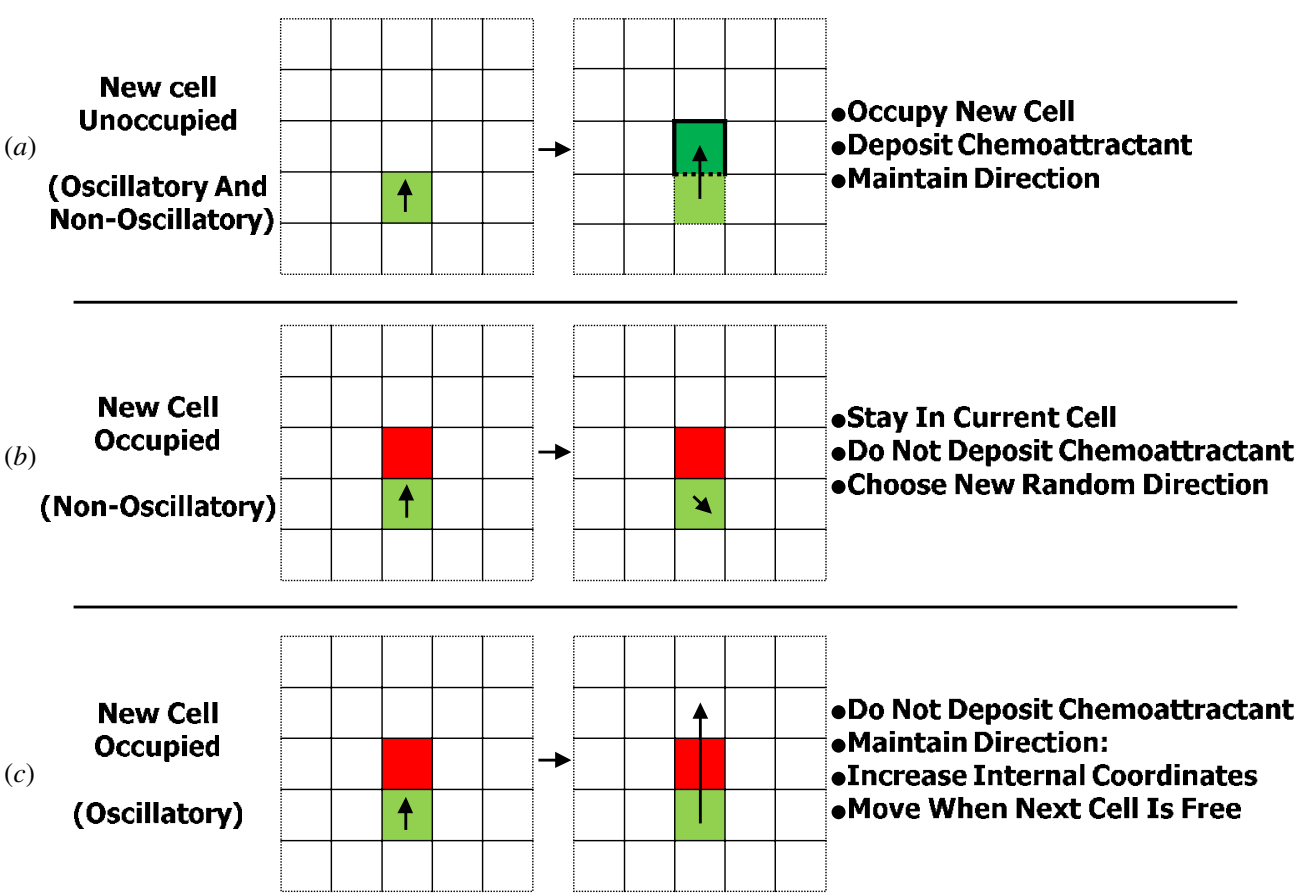

Figure 2. Particle motor behaviour in non-oscillatory and oscillatory conditions. (a) A particle moves forward to occupy a vacant cell and deposits chemoattractant at the new location. (b) A non-oscillatory particle will select a new random location if the preferred cell is occupied. $(c)$ If the preferred cell is occupied, the oscillatory particle will maintain the direction until a cell in front is free.

the chemoattractant. The low-level particle interactions result in complex pattern formation. The population spontaneously forms dynamic transport networks showing complex evolution and quasi-physical emergent properties, including closure of network lacunae, apparent surface tension effects and network minimization. An exploration of the possible patterning parameterization was presented in Jones (2010a). Although the particle model is able to reproduce many of the networkbased behaviours seen in the $P$. polycephalum plasmodium such as spontaneous network formation, shuttle streaming and network minimization, the default motor behaviour does not exhibit oscillatory phenomena and inertial surging movement, as seen in the organism. This is because the default action when a particle is blocked (i.e. when the chosen site is already occupied) is to randomly select a new orientation, resulting in very fluid network evolution resembling the relaxation evolution of soap films and the lipid nanotube networks seen in Lobovkina et al (2008). The oscillatory phenomena seen in the plasmodium are thought to be linked to the spontaneous assembly/disassembly of actomyosin and cytoskeletal filament structures within the plasmodium which generate contractile forces on the protoplasm within the plasmodium. The resulting shifts between gel and sol phases prevent (gel phase) and promote (sol phase) cytoplasmic streaming within the plasmodium. To mimic this behaviour in the particle model requires only a simple change to the motor stage. Instead of randomly selecting a new direction if a move forward is blocked, the particle increments separate internal co-ordinates until the nearest cell directly in front of the particle is free. When a cell becomes free, the particle occupies this new cell and deposits chemoattractant into the lattice (figure 2(c)).
The effect of this behaviour is to remove the fluidity of the default movement of the population. The result is a surging, inertial pattern of movement dependent on population density (the population density specifies the initial amount of free movement within the population). The strength of the inertial effect can be damped by a parameter (pID) which determines the probability of a particle resetting its internal position coordinates, lower values providing stronger inertial movement. When this simple change in motor behaviour is initiated surging movements are seen and oscillatory domains of chemoattractant flux spontaneously appear within the virtual plasmodium showing characteristic behaviours: temporary blockages of particles (gel phase) collapse into sudden localized movement (solation) and vice versa. The oscillatory domains themselves undergo complex evolution including competition, phase changes and entrainment. We utilize these dynamics below to investigate the possibility of generating useful patterns of regular oscillations which may be coupled to provide motive force.

\section{Model setup}

The emergence of oscillatory behaviour in the model corresponds to differences in distribution of protoplasm within the plasmodium and subsequent changes in thickness of the plasmodium. In the P. polycephalum plasmodium, changes in thickness of the plasmodium membrane are used to provide impetus (pumping of material through the vein network, or bulk movement of the plasmodium). There is known to be a reciprocal relationship between the spontaneous contraction of the plasmodium and the subsequent transport of protoplasm away from that region (Takamatsu et al 


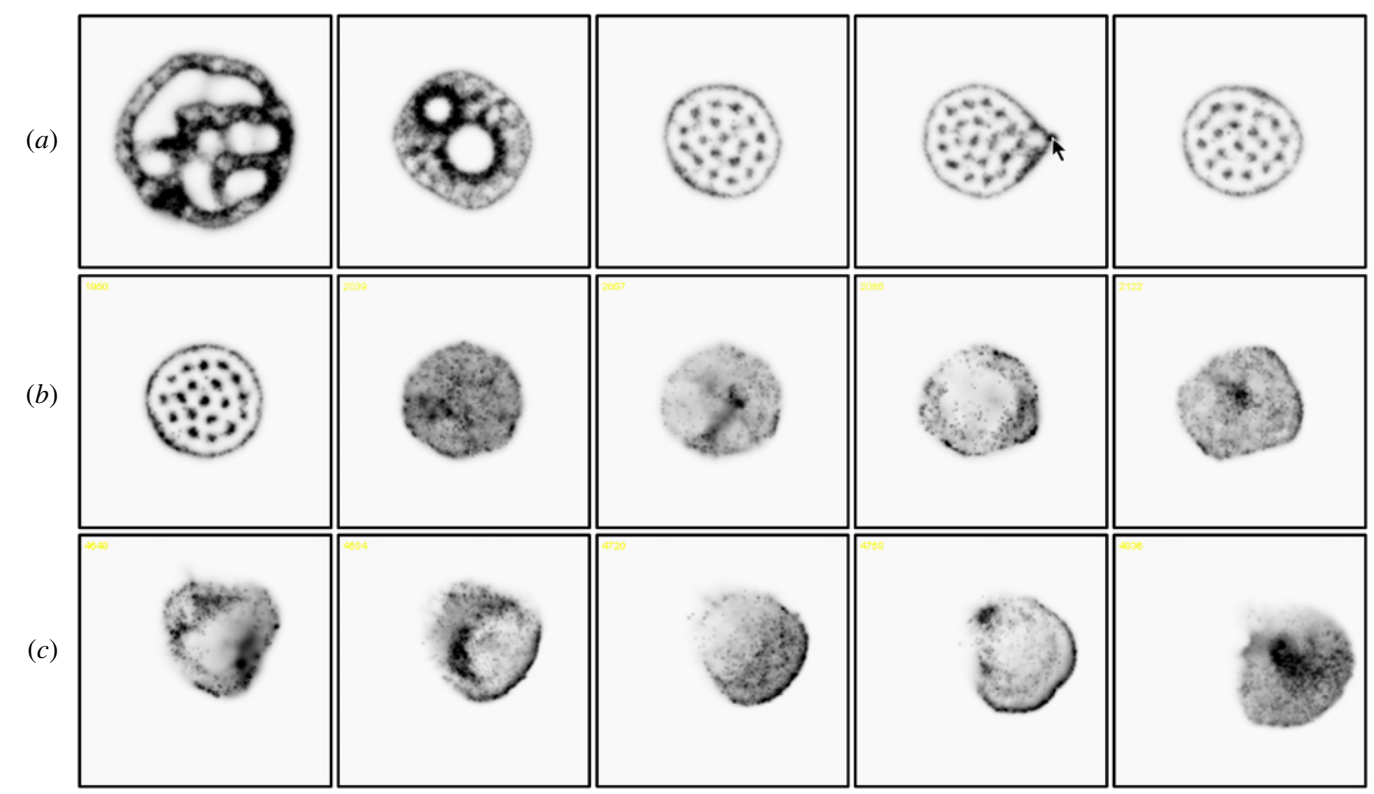

Figure 3. Initiation of oscillatory behaviour and amoeboid movement in simulated plasmodium. Images show flux within simulated plasmodium over time. Images anchored to fixed location. (a) Condensation of blob material in non-oscillatory behaviour shows vacancy domains and resilience to deformation. Collective of 9380 particles, SA90, RA45, SO15. (b) Initial non-oscillatory collective with regular vacancy domains (left) and onset of oscillatory behaviour at 1950, 2039, 2057, 2086 and 2122 scheduler steps. pID set to 0.05 . All other parameters as in $(a)$. (c) Reduction of pID to 0.01 results in stronger oscillations and amoeboid movement at $4648,4684,4720,4768$ and 4836 scheduler steps. All other parameters as in $(a)$.

2000). Thus the region undergoing contraction becomes thinner (allowing more light transmission when illuminated) as protoplasm is being transported away from the contraction zone. Protoplasmic tubes connected to the contraction zone become thicker as more protoplasm is transported from the zone (thus allowing less light transmission). In the computational model the transport of particles represents the free flux of protoplasm within the material and a local increase in flux (particle movement) is indicated in the supplementary video recordings by a temporary increase in greyscale brightness at this location. A decrease in the bulk movement of particles represents local congestion (lack of transport) and is indicated by a decrease in greyscale brightness (since deposition of chemoattractant factor only occurs in the event of successful forward movement). Supplementary video recordings showing the initiation and entrainment of oscillatory phenomena, and recordings from specific figures in the paper text can be found at: http://uncomp.uwe.ac.uk/jeff/collectivemovement.htm. For visual clarity in the static images, the greyscale images are inverted (dark areas indicate greater flux).

The particle population environment is a $2 \mathrm{D}$ lattice, represented by a digitized image configured to represent the habitat of the experimental plasmodium. The habitat is composed of 'wall' regions where particle occupancy and movement cannot occur, 'vacant' regions where occupancy and movement are possible and, where relevant, 'stimulus' regions which provide attraction stimuli, or repulsion stimuli, to the particle population. In the experiments there is an initial period where oscillatory behaviour is not initially activated and this results in self-organized regularly spaced domains within the collective. When oscillatory motor behaviour is induced these regular domains collapse and small domains of disorganized oscillators begin to emerge. The oscillation domains are composed of local areas of flux (brighter) and obstruction (darker). Over time these domains compete and coalesce, causing entrainment of the population into regular oscillation patterns, influenced by both the particle sensory parameters and also by the shape of the experimental arenas.

\section{Emergence of amoeboid movement}

P. polycephalum utilizes internal protoplasmic transport to migrate towards nutrient sources and away from hazardous sources (Durham and Ridgway 1976), and adapts its gross body plan to changing environments (Nakagaki et al 2004, 2007). Small plasmodia can shift the entire plasmodium away from unfavourable conditions such as bacterial or fungal contamination. The plasmodium is also notable for its ability to survive physical damage; fragments of plasmodium excised can survive independently and individual plasmodia may be fused to form a single organism. To utilize a vehicular analogy, $P$. polycephalum not only represents the internal mechanicals (motive force mechanism, transmission coupling), but also the moving vehicle itself, and is a vehicle which can survive the removal of parts, the introduction of new foreign parts and the repair of damaged parts.

We set out to explore the behaviour of the particle collective to assess its behaviour when compared to a fragment of $P$. polycephalum plasmodium. When oscillatory motor behaviour is not used the particle collective condenses into a uniform circular shape as the initial transport network condenses (figure 3(a)). The non-oscillatory blob shows regular vacancy domains (dark areas) and the fluid particle 


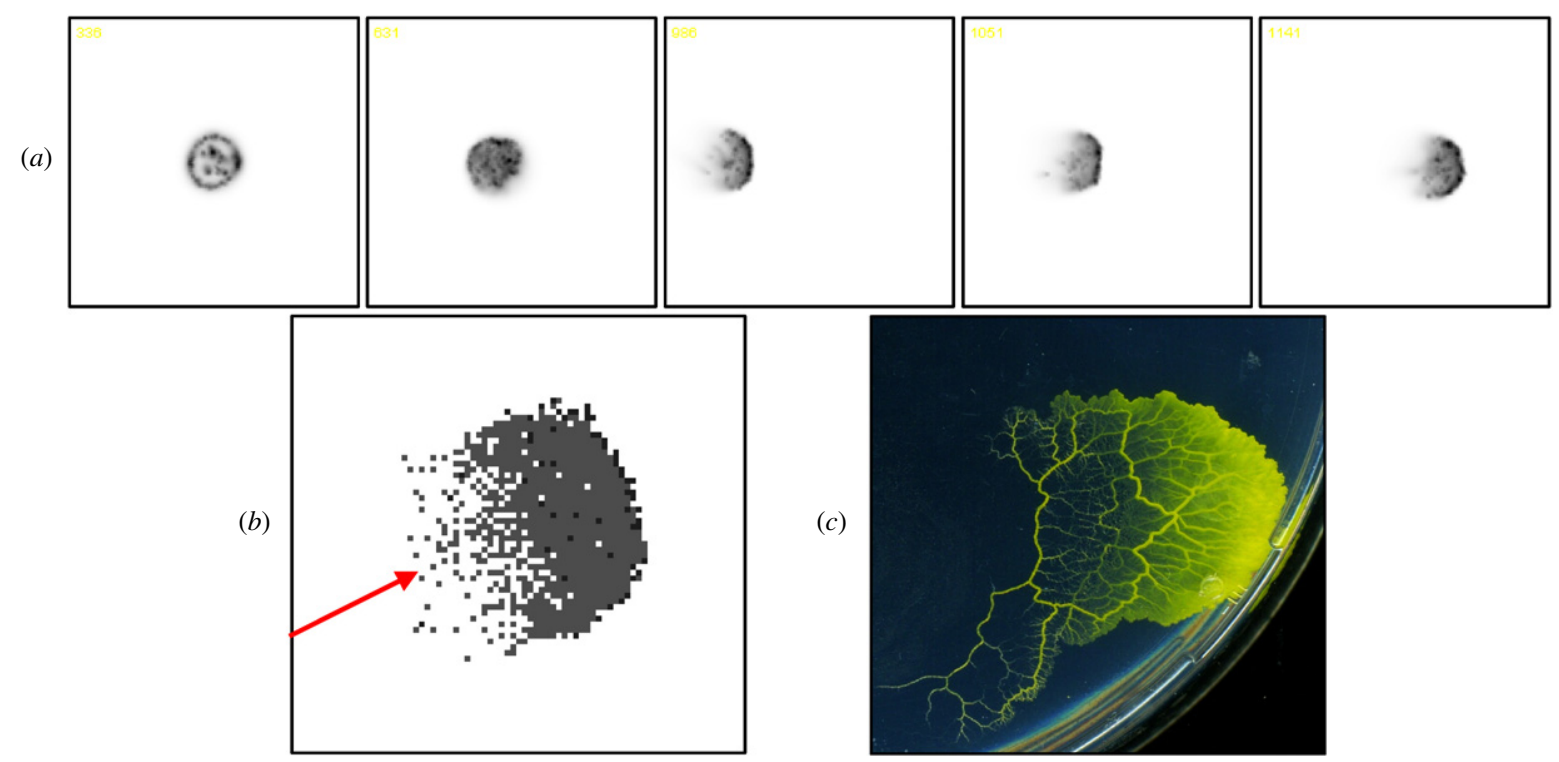

Figure 4. Persistent forward movement of blob fragments in small simulated plasmodia. Images show flux within simulated plasmodium fragments over time. Images anchored to fixed location. (a) Non-oscillatory condition, initiation of oscillatory behaviour and (final three images) self perpetuating transport of blob fragment. Chemoattractant flux concentration is greatest at the front of the dome shape.

(b) Enlargement showing particle composition of the moving blob fragment. The persistent shape is maintained despite repeated turnover of component parts. Population size 900 particles, SA90, RA45, SO9, pID 0.001. (c) Independently moving small plasmodial fragment of P. polycephalum.

motion afforded by the non-oscillatory motor condition ensures that the blob is cohesive and takes a minimal shape. The non-oscillatory blob is also resilient to external perturbation. When excited by an externally applied source of chemoattractant (figure 3(a), mouse position in fourth image), the deformation of the collective induced by the stimulus as it is attracted to the stimuli is repaired when the stimulus is removed, the collective returning to its minimal shape.

When oscillatory motor behaviour is initialized the regular domains collapse as the particle motion becomes less fluid (figure $3(b)$ ) and oscillations travel through the collective. Because the small collective is not constrained by any externally applied pattern the oscillations distort the shape of the collective. When the pID parameter is further reduced to 0.01 there is even greater restriction on the fluidity of individual particle movement and the oscillations become stronger and distort the collective's boundary significantly. The large shift of a mass of particles causes the collective to move across its environment. The cohesion of the collective is maintained but other SA/RA parameter settings, combined with lower sensor interaction ( $\mathrm{SO}$ ) distance, can result in the fragmentation of the collective (see supplementary material for examples of oscillation patterns using different SA/RA combinations).

\section{Persistent movement in a small blob fragment}

The amoeboid movement seen in figure 3 occurs because the oscillation waves distort the boundary of the collective whilst it is still able to maintain a cohesive whole. Because the population maintains its cohesion, any distortion of the boundary on one side must result in a shift in population distribution from the opposite side (since the collective is non-compressible and occupies a fixed area). The diameter of the collective (a function of the number of pixel sized particles comprising it) must be large enough to for oscillations to emerge and to confine an oscillation pattern within it.

When the collective is comprised of only a relatively small number of particles, the distortion of the boundary forms an approximately semicircular domed shape and the small number of particles ensures that the collective cannot maintain a fully circular shape. However the persistence of forward movement generated by the oscillatory motor behaviour at low pID values causes the dome shape itself to be maintained over time, and the small blob fragment is able to move forwards (figure 4(a)). Movement of the fragment is relatively smooth and different from the pulsatile motion observed in larger collectives. Particles move towards the front of the domed profile (figure $4(b)$, dark pixels) and then, over time, move to the side. Particles at the sides of the fragment ultimately fall behind only to re-enter the dome at the centre. Higher pID values result in more frequent changes of direction of the fragment as the dome shaped front profile cannot be maintained. If the population size is increased, the single sided dome shape cannot be maintained and the resultant motion becomes pulsatile and chaotic. The movement of small blob fragments in the particle collective mimics amoeboid motion observed in $P$. polycephalum on a non-nutrient substrate (figure $4(c)$ ) which has been shown to be equivalent to the propagation of wave fragments in sub-excitable media (Adamatzky et al 2008, Adamatzky 2010b). Both artificial and real blobs exhibit reflection (reversion of direction) when encountering the boundary of their environment and can both be directionally guided by the placement of attractants and repellents (Adamatzky 2010a). 


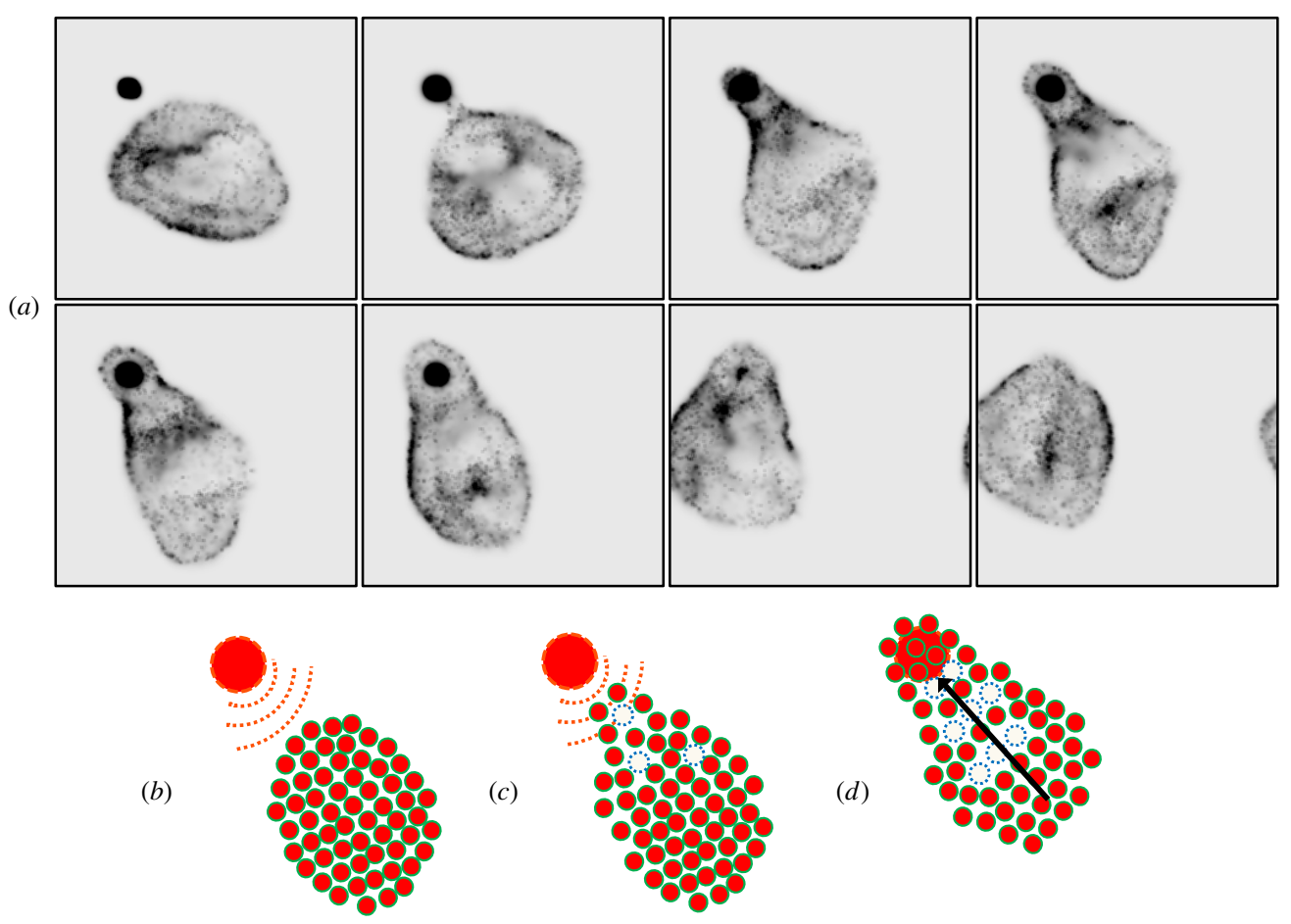

Figure 5. Control of amoeboid movement by attraction. Images show flux within simulated plasmodium over time. Images anchored to fixed location. (a) A persistent source of chemoattractant is placed into the diffusion field which provokes the extension of the collective. The collective engulfs the source by moving via travelling waves towards the source. When the source is exhausted the collective re-adopts its original approximately circular shape. (b) Schematic illustration of attraction of the collective by the nutrient source. (c) Migration of leading particles towards the source. $(d)$ Emergence of travelling waves pulling the collective to engulf the source.

\section{External influence of collective movement-attraction and repulsion}

Movement of the $P$. polycephalum plasmodium is strongly affected by local environmental conditions. Attractant sources (such as increasing temperature gradients and chemoattractant nutrients) cause the plasmodium to move and grow towards the attractants whereas repulsive sources (salts, dry regions) cause the plasmodium to try to avoid such regions (Durham and Ridgway 1976). The plasmodium is able to integrate many separate localized inputs to compute its response to the environment. One method in which this is achieved is by the modulation of local oscillation patterns in response to attractants or hazards - attractants tend to increase localized oscillation strength and hazards decrease oscillation strength. We set out to see if a localized response to external influences could be used to govern the collective movement of the particle population.

Attractant sources were previously used as a method to confine the collective to a region by pinning it down. By externally presenting an attractant source (effectively a simulated nutrient source) near to a cohesive blob of virtual material (circle in figure 5(a)) a concentration gradient emerged from the source (figure $5(b)$ ). When the diffusion gradient reached the sensors of the closest particles at the front of the collective it provoked local movement towards the source. The cohesion within the collective resulted in a pseudopodium-like extension of the border region which extended towards the source (figure 5(c)), ultimately engulfing it. Travelling waves spontaneously emerged within the collective which were directed at the source, causing the collective to shift its position towards the source (figure $5(d)$ ). Consumption of the source was simulated by simply decrementing the value projected to the diffusion field when the source was covered by a particle. When the source was consumed by the population, the collective regained its previous approximately circular shape.

To approximate the repulsion of the collective to hazardous sources such as the simulated response to irradiation by visible light we added a condition to the sensory stage of the algorithm to the effect that if any particles of the collective were in a region exposed to 'light' (a defined area within the arena), those particles would have their sensitivity to chemoattractant diminished whilst they remained in this region (this achieved by multiplying the sampled sensor values with a weighting factor less than 1 , lower values generating a stronger response to irradiation). The effect of exposing regions of the collective to simulated light damage was that the collective immediately started to move away from the irradiated region (figure 6(a)). Specifically, oscillation waves moved from the irradiated region towards the unexposed regions. The shift of particles from the irradiated region eventually moved the collective away from the stimulus. The cause of movement away from the light can be found at the interface between irradiated and unexposed areas. Before irradiation (figure $6(b)$ ) all regions of the collective are equally attractive to the particles (subject to fluctuations caused by discrepancies in particle movement and intrinsic oscillations within the collective). There is a strong coupling between the particles in the collective caused 
(a)

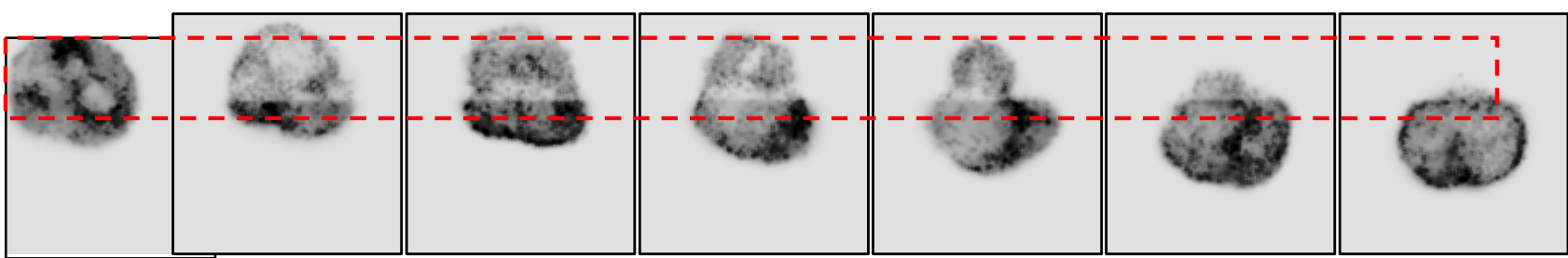

(b)

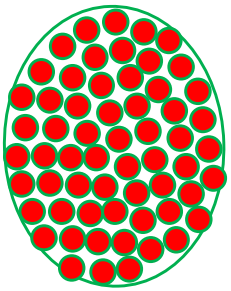

(c)

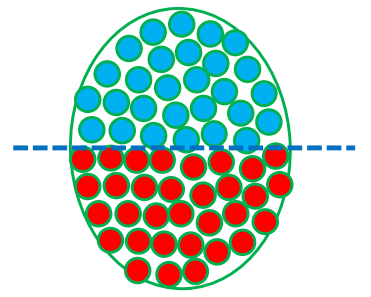

(d)

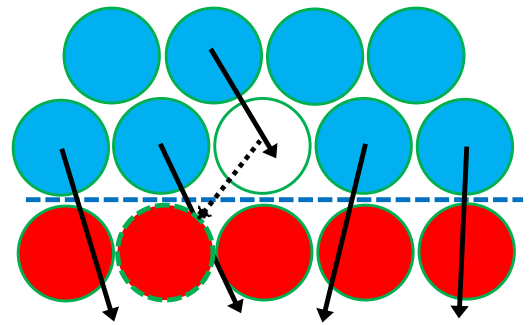

Figure 6. Avoidance of simulated light irradiation by particle collective. Images show flux within simulated plasmodium over time. Images anchored to fixed location. (a) Area within dashed box is stimulated with simulated light irradiation. Particle collective oscillates sending travelling waves towards the region which is unexposed, moving the collective away from the irradiated region. (b) Schematic illustration of condition before irradiation - all particles sense equal concentration of chemoattractant. (c) Irradiated areas (top) are perceived as weaker concentration. $(d)$ Particles at the irradiation interface are more attracted to unexposed areas. Migration across interface causes chemoattractant deposition, causing further attraction to region and vacant space.

(a)
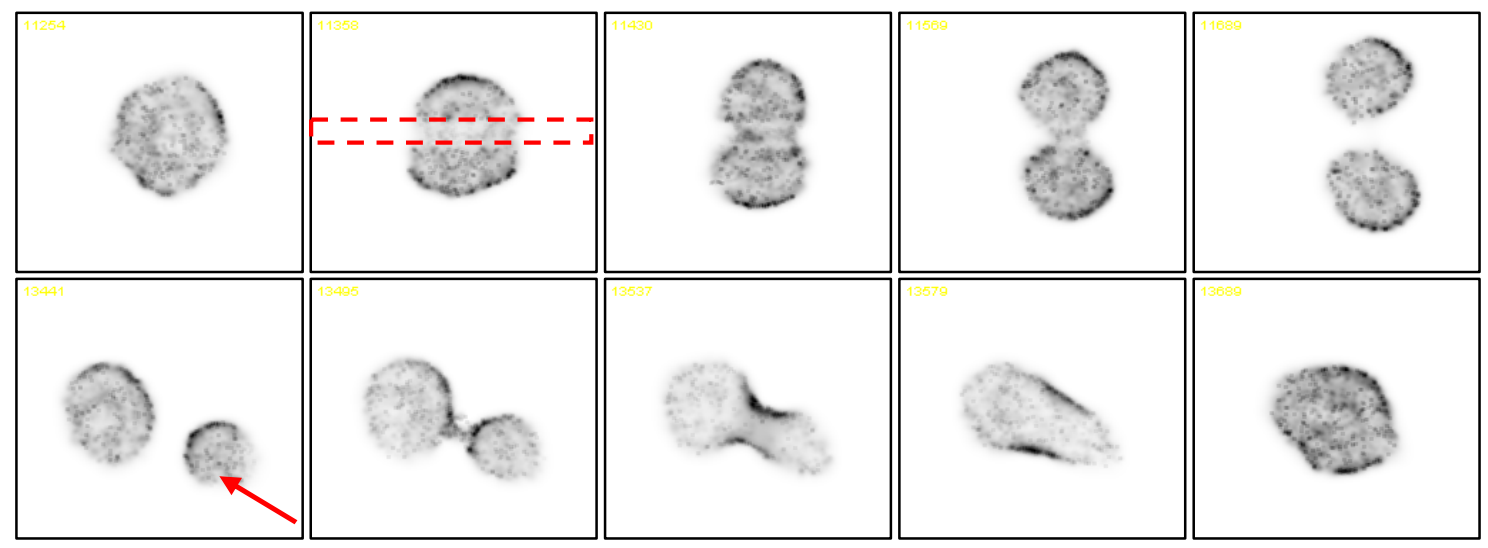

$(c)$

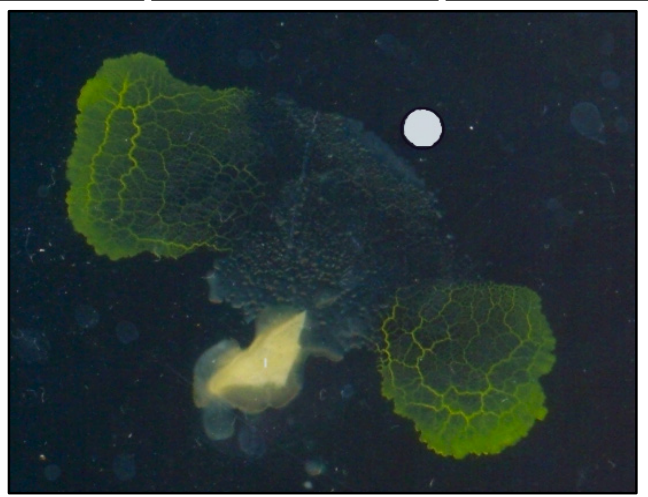

Figure 7. Controlling blob morphology by splitting and fusion. Images show flux within simulated plasmodium over time. Images anchored to fixed location. (a) 'Blob' of aggregate particles is split by applying simulated light irradiation (dashed box), disrupting particle flux in that region. The single blob separates into two smaller blobs, each capable of individual oscillation and external control. $(b)$ Fusion of two independent particle aggregates. The blob on the lower right is guided diagonally upwards in the direction of the arrow towards the larger blob. The two independent blobs fuse forming a single aggregate. $(c)$ Cleavage of plasmodium of $P$. polycephalum. Fragment of plasmodium initialized at triangular region. Crystal of potassium chloride placed at circular region. Diffusion of potassium chloride cleaves the plasmodium into two independent fragments.

by the offset sensor distance. Some of the particles at the interface of the irradiated region will receive input from the unexposed region and will be attracted to that area because the chemoattractant concentration in unexposed areas is perceived as greater due to the damping in irradiated regions (figure 6(c)). The movement of particles near the interface towards unexposed regions causes both new vacant spaces (figure $6(d)$ ) and also an increase in 
(a)
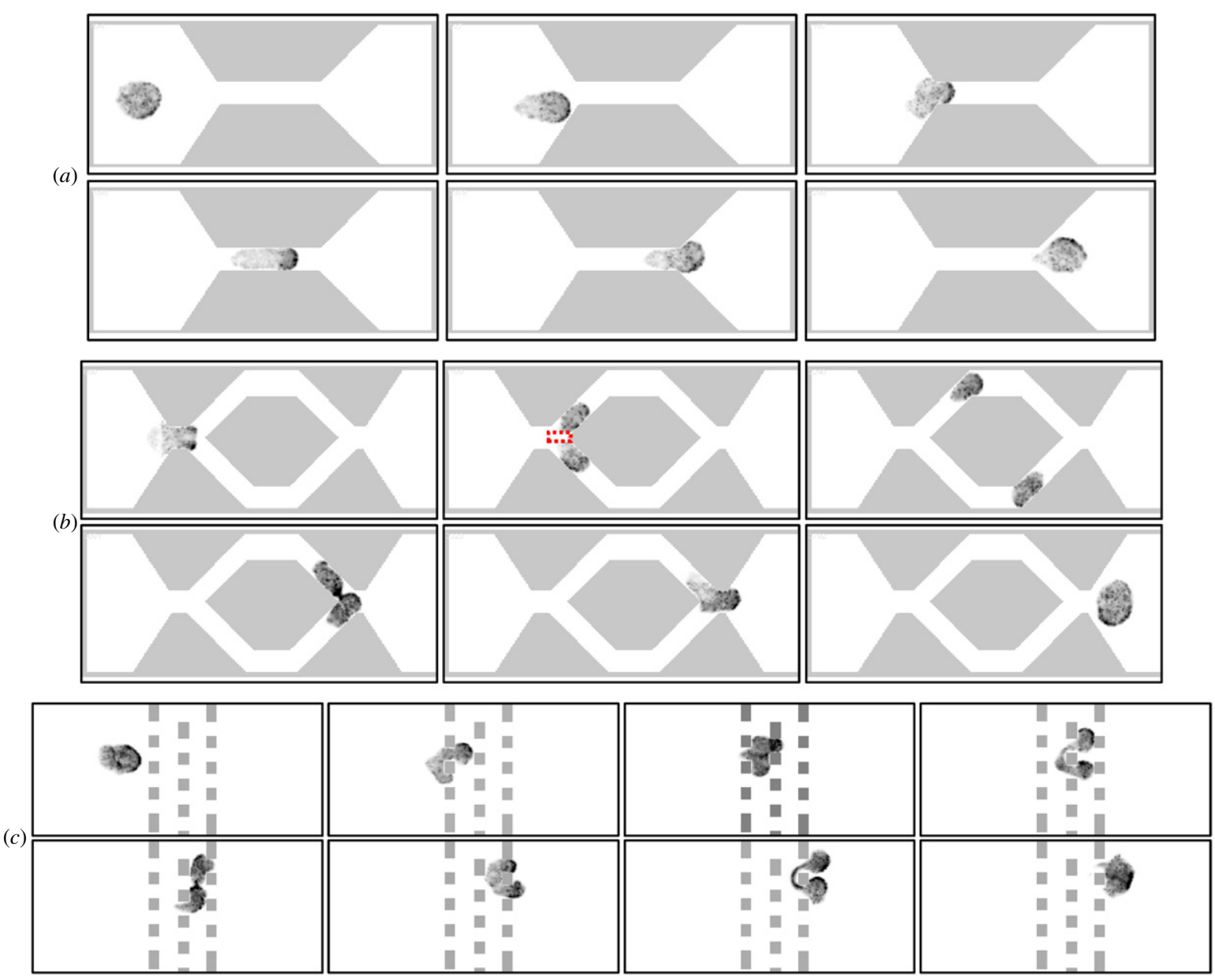

Figure 8. Exploiting the adaptive morphology of the collective. Aggregate blob of simulated plasmodium is pushed by the application of external simulated light irradiation (illuminated at the left side of the aggregate, not shown). (a) Large aggregate moves through a channel narrower than the aggregate diameter. (b) Aggregate is cleaved by hazard irradiation (boxed) and guided separately before being re-fused into original size. (c) Aggregate is pushed through tortuous narrow grating and re-forms original aggregate shape.

chemoattractant concentration (because only mobile particles deposit chemoattractant). This results in a further increased attraction to the interface region, until eventually the entire collective has migrated from the irradiated region.

\section{Morphological adaptation of the collective}

The previous results demonstrated that the collective changes its shape during self-oscillatory behaviour and also in response to simulated attractants and hazards. The collective retains its typically circular shape due to the cohesion of the individual particles making up the population. When the morphology of the collective is disturbed by its movement towards, or away from, externally applied stimuli it can re-form the original shape when the stimulus is removed. An adaptive morphology is a very desirable property in robotic devices since it imbues the robot with great flexibility of size and movement, enabling it to traverse environments which traditionally are difficult to navigate (for example narrow spaces, gratings, etc). This feature is only possible because the properties of the movement and guidance of the collective are distributed throughout the collective and not located in fixed sized and inflexible units as is the case with conventional robotic systems. This is also the case with the $P$. polycephalum plasmodium which adapts its shape and growth patterns in response to its environment. One of the most remarkable properties of the plasmodium is the ability to survive external damage beyond simple attraction and repulsion. A piece of plasmodium excised from the growing tip can survive, and indeed continue to move and grow as an independent entity. Furthermore two independent plasmodia can fuse to form a single plasmodium when placed in close proximity. These phenomena are not only desirable from a robotics perspective in terms of resilience and damage repair, but offer new and as yet little explored opportunities in robotic movement and control.

We set out to find if the particle collective could also replicate these highly desirable features as seen in the real plasmodium. We took a large single oscillating collective (5000 particles) and applied a narrow band of hazardous simulated light irradiation through its centre (figure $7(a)$, dashed box represents irradiated area). Particles immediately began to surge away from the irradiated region on both sides and the collective narrowed in diameter and became further pinched in shape until the collective was cleaved into two independently controllable blobs. The cleavage mechanism can be applied in different ways. For example both resulting 
blobs can be of equal size and have similar oscillatory properties. Alternately it is possible to cleave the collective in such a way to have one large stationary blob and one smaller mobile blob (recall that a blob which is small enough will be able to move spontaneously in a persistent direction).

It is possible to guide each blob independently using either a pulling type mechanism (externally applied attractants) or a pushing type mechanism (simulated irradiation). In figure $7(b)$ we guide the lower right blob (arrowed) towards the larger blob by pushing it from its opposite side with simulated irradiated light. As the blobs become closer (specifically, to a separation distance which is sufficient for the border particles in each blob to sense the chemoattractant flux in the other blob) the closest border regions of each blob surge towards each other and a single larger collective is formed by the fusion. Cleavage and guidance of the particle collective by simulated hazards reproduces the control of $P$. polycephalum plasmodial migration (figure 7(c)) by repellents (Adamatzky 2010a).

As an example of the robotic flexibility endowed by the adaptive morphology of the collective, and the guidance mechanisms enabled by its external control, we show in figure 8 three examples of how a blob can be guided externally (in this case a repulsive pushing by simulated irradiation) in order to traverse narrow, separated or tortuous paths where the path may be much narrower than the diameter of the collective itself. In figure $8(a)$, the blob has a diameter of approximately 52 pixels and is guided through a channel of width 30 pixels by automatically reconfiguring its morphology. In figure $8(b)$, the blob is split into two parts by irradiating a rectangular region at the entrance of the channel. The two separated blobs may be guided separately by simulated radiation and are refused near the exit of the channels. Finally, in figure 8(c), the blob automatically separates its structure in response to the different obstacles and re-forms its shape by mutual attraction and cohesion of the particles. No fine control of the individual components, nor complex pre-determinism of path choice, is necessary; the collective is guided by simple avoidance of the simulated irradiation (the irradiation location is not shown, but follows the previous examples of simply exposing the rearward part of the collective to push it forwards). When clear of the obstacles, the original circular shape is re-formed. If the blob is returned backwards through the obstacle path again the path chosen can be somewhat different to the original path with the same result.

\section{Conclusion and discussion}

We have examined the problem of generating collective amoeboid movement in a multi-agent approximation of the plasmodium of true slime mould $P$. polycephalum using selforganization between simple non-oscillatory component parts. $P$. polycephalum is attractive because it satisfies many physical and computational properties which are desirable in robotics applications (self-oscillatory, simple components, distributed sensory and motor control, integration of multiple sensory stimuli, amorphous and adaptive shape, amenable to external influence, resilience to damage, self repair) and can be regarded as a living example of a so-called smart material because of the way in which it combines robotic and control functions in a distributed manner throughout its constituent material.

How can such complex behaviour emerge from the interactions between simple component parts? Answering this question may provide insights into the development of non-living smart materials which would have the advantages demonstrated by the $P$. polycephalum plasmodium, but without some of its limitations, such as slow speed, fragility and unpredictability. Because of its simple components and structure, any search for a 'secret source' of the plasmodium's complexity would be fruitless. Instead we set about answering the question by posing in reverse: rather than try to find out how the organism produces such complex behaviour from simple parts, is it possible to artificially generate similarly complex behaviour from simple parts and interactions? We utilized a previous particle model of emergent transport networks where a collective of identical particles with identical behaviour was used to construct and minimize synthetic and emergent transport networks. We modified its motor algorithm in a very simple way so that, instead of smooth network flow, we obtained a more resistant flow of particles. The addition of transient resistance to particle flux was sufficient to generate complex oscillatory behaviour strongly similar to that observed in the plasmodium itself.

The condensation of the collective in its virtual environment resulted in cohesive blob-like sheets of virtual material held together by cohesion which exhibited spontaneous internal oscillations. The oscillations were composed of regular spatial domains of mobile particles and temporarily restricted particles. The wave-like propagation of the oscillatory domains resulted in a collective amoeboid movement as the perimeter of the particle collective was deformed by the internal oscillations. External control of the blob sheets was achieved by stimulating the collective with simulated attractants (pulling the collective towards the source) and repellents ('pushing' the collective away from simulated light irradiation). We were also able to reproduce the resilience of the $P$. polycephalum plasmodium to damage by cleaving the collective into two separate and independent blobs and fusing two independent blobs to form a single functional blob. Finally the adaptive morphology of the collective was demonstrated by guiding the collective through an obstacle field narrower than the diameter of the collective itself. The flexible modularity and morphology seen in this approach builds upon previous work on the external influence and control of robotic devices (Bojinov et al 2002, Salemi et al 2001) by enabling separate and functional modules of arbitrary size to be created and re-formed from a single collective.

The results demonstrate how very simple and local low-level interactions within a simple material can generate complex and emergent behaviour which appear to transcend the capabilities of the simple matter of which they are composed. As noted by previous swarm approaches, there need not be anything magical or special about the properties of these materials; the complexity emerges merely from their interactions (Buhl et al 2006, Reynolds 1987, Vicsek et al 1995). This complex behaviour is harnessed effortlessly by organisms such as $P$. polycephalum as part of a parsimonious 
survival strategy, enabling their persistence in unpredictable, changeable and hazardous environmental conditions. By understanding the generative mechanisms underlying the complex behaviour it may be possible to incorporate these features within real physical materials for small scale robotic devices. By utilizing the robotic substrate material itself for distributed computation, transport and movement it may be possible to reduce the total number of component parts and also reduce the number of different types of components, thus further simplifying the production of the devices.

\section{Acknowledgment}

The work was partially supported by the Leverhulme Trust research grant F/00577/1 'Mould intelligence: biological amorphous robots'.

\section{Q2 References}

Adamatzky A 2007 Physarum machines: encapsulating reaction-diffusion to compute spanning tree Naturwissenschaften 94 975-80

Adamatzky A 2008 Developing proximity graphs by Physarum polycephalum: does the plasmodium follow Toussaint hierarchy? Parallel Process. Lett. 19 105-27

Adamatzky A 2010a Routing Physarum with repellents Eur. Phys. J. E 31 403-10

Adamatzky A 2010b If BZ medium did spanning trees these would be the same trees as Physarum built Phys. Lett. A 373 952-6

Adamatzky A 2010c Manipulating substances with Physarum polycephalum Mater. Sci. Eng. C 30 1211-20

Adamatzky A, De Lacy Costello B and Shirakawa T 2008 Universal computation with limited resources: Belousov-Zhabotinsky and Physarum Computers Int. J. Bifurcation Chaos $182373-89$

Adamatzky A and Jones J 2008 Towards Physarum robots: computing and manipulating on water surface J. Bionic Eng. 5 348-57

Ben-Jacob E 2003 Bacterial self-organization: co-enhancement of complexification and adaptability in a dynamic environment Phil. Trans. R. Soc. A 361 1283-312

Bojinov H, Casal A and Hogg T 2002 Multiagent control of self-reconfigurable robots Artif. Intell. 142 99-120

Buhl J, Sumpter D J T, Couzin I D, Hale J J, Despland E, Miller E R and Simpson S J 2006 From disorder to order in marching locusts Science 3121402

Carlile M J 1970 Nutrition and chemotaxis in the myxomycete Physarum polycephalum: the effect of carbohydrates on the plasmodium J. Gen. Microbiol. 63221

Dreyfus R, Baudry J, Roper M L, Fermigier M, Stone H A and Bibette J 2005 Microscopic artificial swimmers Nature 7060862

Durham A C and Ridgway E B 1976 Control of chemotaxis in Physarum polycephalum J. Cell Biol. 69 218-23

Goldstein S C, Mowry T C, Campbell J D, Ashley-Rollman M P, De Rosa M, Funiak S, Hoburg J F, Karagozler M E, Kirby B and Lee P 2009 Beyond audio and video: using claytronics to enable pario AI Mag. 3029

Helbing D 2001 Traffic and related self-driven many-particle systems Rev. Mod. Phys. 73 1067-141

Helbing D, Molnar P, Farkas I J and Bolay K 2001 Self-organizing pedestrian movement Environ. Plan. B 28 361-84

Helbing D, Molnar P and Schweitzer F 1998 Computer simulations of pedestrian dynamics and trail formation arXiv preprint cond-mat/9805074
Jones J 2010a Characteristics of pattern formation and evolution in approximations of physarum transport networks Artif. Life 16 127-53

Jones J 2010b The emergence and dynamical evolution of complex transport networks from simple low-level behaviours Int. J. Unconv. Comput. 6 125-44

Jones J and Adamatzky A 2010 Towards physarum adders BioSystems 101 51-8

Kennedy B, Melhuish C and Adamatzky A (eds) 2001 Biologically inspired robots (SPIE Press)

Kishimoto U 1958 Rhythmicity in the protoplasmic streaming of a slime mould, Physarum polycephalum J. Gen. Physiol. 41 1223-44

Kube C R and Bonabeau E 2000 Cooperative transport by ants and robots Robot. Auton. Syst. 30 85-101

Lagzi I, Soh S, Wesson P J, Browne K P and Grzybowski B A Maze solving by chemotactic droplets J. Am. Chem. Soc. 132 1198-9

Lobovkina T, Dommersnes P G, Tiourine S, Joanny J F and Orwar O 2008 Shape optimization in lipid nanotube networks Eur. Phys. J E 26 295-300

Lobovkina T, Gözen I, Erkan Y, Olofsson J, Weber S G and Orwar O 2009 Protrusive growth and periodic contractile motion in surface-adhered vesicles induced by $\mathrm{Ca}^{2+}$-gradients Soft Matter 6 268-72

Matsushita M, Wakita J, Itoh H, Watanabe K, Arai T and Matsuyama T 1999 Formation of colony patterns by a bacterial cell population Physica A 274

Nakagaki T, Kobayashi R, Nishiura Y and Ueda T 2004 Obtaining multiple separate food sources: behavioural intelligence in the Physarum plasmodium Proc. R. Soc. B 271 2305-10

Nakagaki T, Saigusa T, Tero A and Kobayashi R 2007 Effects of amount of food on path selection in the transport network of an amoeboid organism (World Scientific) p 94

Nakagaki T, Umemura S, Kakiuchi Y and Ueda T 1996 Action spectrum for sporulation and photoavoidance in the plasmodium of Physarum polycephalum, as modified differentially by temperature and starvation Photochem. Photobiol. 64 859-62

Nakagaki T, Yamada H and Toth A 2000a Maze-solving by an amoeboid organism Nature $\mathbf{4 0 7}$

Nakagaki T, Yamada H and Ueda T 2000b Interaction between cell shape and contraction pattern in the Physarum plasmodium Biophys. Chem. 84 195-204

Reynolds C 1987 Flocks, herds and schools: a distributed behavioral model ACM SIGGRAPH Computer Graphics vol $21 \mathrm{pp} 25-34$

Rus D and Vona M 2000 A physical implementation of the self-reconfiguring crystalline robot IEEE vol 2 pp 1726-33

Saga N and Nakamura T 2004 Development of a peristaltic crawling robot using magnetic fluid on the basis of the locomotion mechanism of the earthworm Smart Mater. Struct. 13566

Saigusa T, Tero A, Nakagaki T and Kuramoto Y 2008 Amoebae anticipate periodic events Phys. Rev. Lett. 10018101

Salemi B, Shen W M and Will P 2001 Hormone-controlled metamorphic robots IEEE vol 4 pp 4194-9

Shirakawa T, Adamatzky A, Gunji Y and Miyake Y 2009 On simultaneous construction of Voronoi diagram and Delaunay triangulation by Physarum polycephalum Int. J. Bifurcation Chaos 19 3109-17

Shirakawa T and Gunji Y 2010 Computation of Voronoi diagram and collision-free path using the plasmodium of Physarum polycephalum Int. J. Unconv. Comput. $679-89$

Stoy K 2006 Using cellular automata and gradients to control self-reconfiguration Robot. Auton. Syst. 54 135-41

Suh J W, Darling R B, Bohringer K F, Donald B R, Baltes H and Kovacs G T 2000 Fully programmable MEMS ciliary actuator arrays for micromanipulation tasks IEEE vol 2 pp 1101-8 
Takamatsu A, Fujii T and Endo I 2000 Control of interaction strength in a network of the true slime mold by a microfabricated structure BioSystems 55 33-8

Trimmer B A, Takesian A E, Sweet B M, Rogers C B, Hake D C and Rogers D J 2006 Caterpillar locomotion: a new model for soft-bodied climbing and burrowing robots vol $1 \mathrm{pp} 1-10$

Tsuda S, Aono M and Gunji Y 2004 Robust and emergent Physarum logical-computing BioSystems 73 45-55

Tsuda S, Zauner K and Gunji Y 2007 Robot control with biological cells BioSystems 87 215-23

Ueda T, Terayama K, Kurihara K and Kobatake Y 1975 Threshold phenomena in chemoreception and taxis in slime mold Physarum polycephalum J. Gen. Physiol. 65223
Umedachi T, Kitamura T, Takeda K, Nakagaki T, Kobayashi R and Ishiguro A 2009 A modular robot driven by protoplasmic streaming Distrib. Auton. Robot. Syst. 8 193-202

Vicsek T S, Czirók A S, Ben-Jacob E, Cohen I and Shochet O 1995 Novel type of phase transition in a system of self-driven particles Phys. Rev. Lett. 75 1226-9

Yokoi H, Nagai T, Ishida T, Fujii M and Iida T 2003 Amoeba-like robots in the perspective of control architecture and morphology/materials Morpho-Functional Machines: The New Species (Tokyo: Springer) pp 99-129

Zhang L, Abbott J J, Dong L, Kratochvil B E, Bell D and Nelson B J 2009 Artificial bacterial flagella: fabrication and magnetic control Appl. Phys. Lett. 94064107

Zhang S 2003 Fabrication of novel biomaterials through molecular self-assembly Nature Biotechnol. 21 1171-8 


\section{QUERIES}

\section{Page 1}

Q1

Author: Please be aware that the colour figures in this article will only appear in colour in the Web version. If you require colour in the printed journal and have not previously arranged it, please contact the Production Editor now.

\section{Page 11}

Q2

Author: Please check the details for any journal references that do not have a blue link as they may contain some incorrect information. Pale purple links are used for references to arXiv e-prints.

Q3

Author: Please check whether the journal name is okay as set in reference 'Ben-Jacob (2003)'.

Q4

Author: Please provide the name of book and the place of publication in reference 'Kennedy et al (2001)'.

Q5

Author: Please provide page range in references 'Matsushita et al (1999)' and 'Nakagaki et al (2000a)'.

Q6

Author: Please provide the name of book and the place of publication in reference 'Nakagaki et al (2007)'.

Q7

Author: Please provide conference title in references 'Rus, Vona (2000)', 'Salemi et al (2001)', 'Suh et al (2000)' and 'Trimmer et al (2006)'. 